\title{
Genetic Variability of RXRB, PPARA, and PPARG in Wegener's Granulomatosis
}

\author{
Stefan Wieczorek, ${ }^{1}$ Silvia Knaup, ${ }^{1}$ Wolfgang L. Gross, ${ }^{2}$ and Jörg T. Epplen ${ }^{1}$ \\ ${ }^{1}$ Human Genetics Department, Ruhr University, 44801 Bochum, Germany \\ ${ }^{2}$ Department of Rheumatology, University Hospital Lübeck and Klinikum Bad Bramstedt, 24576 Bad Bramstedt, Germany
}

Correspondence should be addressed to Stefan Wieczorek, stefan.wieczorek@rub.de

Received 29 August 2008; Revised 4 December 2008; Accepted 5 January 2009

Recommended by Mostafa Badr

A major genomic region involved in Wegener's granulomatosis includes the gene for retinoid receptor beta $(R X R B)$ which forms heterodimers with peroxisome proliferator-activated receptors (PPARs). It is unclear whether this association directly arises from the $R X R B$ allele(s) or via a linked variation. In order to reveal any hitherto unknown and potentially disease-relevant variation of the RXRB gene, we have genotyped four tagging SNPs of this genomic region and have directly sequenced selected WG patients and controls representing disease-associated haplotypes. Additionally, we have genotyped 2 SNPs each in the genes for PPAR $\alpha$ and PPAR $(P P A R A$ and PPARG). Hence, we confirmed the strong association of the RXRB locus with WG but could not reveal any novel variation in $R X R B$. None of the PPARA and PPARG SNPs showed association with WG. Moreover, no epistatic effect was seen between $R X R B$ and PPARA/PPARG alleles. These results do not support an etiopathological role of PPAR in WG. Analyses of further genes functionally linked to RXRB may provide additional data useful to evaluate the $R X R B$ association found in WG.

Copyright (c) 2009 Stefan Wieczorek et al. This is an open access article distributed under the Creative Commons Attribution License, which permits unrestricted use, distribution, and reproduction in any medium, provided the original work is properly cited.

\section{Introduction}

Wegener's granulomatosis (WG) is a form of small vessel vasculitis belonging to the group antineutrophil cytoplasmic antibody- (ANCA-) associated vasculitides (AAV). WG usually presents with granulomatous lesions of the upper respiratory tract and often develops into generalized vasculitis with multiple organ involvement in later disease stages. In WG ANCA typically recognize proteinase 3 , delineating this diagnostic entity from other AAV forms like Churg Strauss syndrome or microscopic polyangiitis, in which ANCA are usually directed against myeloperoxidase (reviewed in [1]). Current concepts of its etiopathology are incomplete, but WG is accounted to the large and heterogenous group of complex disorders arise from a mostly elusive interplay of environmental and genetic factors.

A major genomic locus for WG was identified on chromosome 6p21.3, including (among others) the genes for HLA-DPB1, RXRB, and RING1 [2]. The linkage disequilibrium (LD) pattern of this genomic region is complex with larger blocks of strong LD alternating with regions of high recombination rates. There is evidence that the association of this locus with the disorder may arise from more than one variation, making $6 \mathrm{p} 21.3$ a quantitative trait locus for WG. While a strong association is demonstrable with the $H L A-D P B 1^{*} 0104$ allele, a (partly) independent association was detected for the defined region including potential WG candidate genes RING1 and RXRB [3]. In addition certain alleles of $R X R B$, the gene encoding retinoid $\mathrm{X}$ receptor beta, are highly significantly associated with WG susceptibility [4].

Peroxisome proliferator-activated receptors (PPAR) are group of nuclear receptors mediating the effects of peroxisome proliferators on gene transcription. Binding of PPAR to regulatory DNA sequences requires the heterodimerization with RXR. PPAR mediated effects are complex and include modulation of inflammatory processes. PPAR $\alpha$ is a ligand for leukotriene B4, and PPAR $\alpha$ deficient mice show a prolonged response to inflammatory stimuli [5]. PPAR $\alpha$ also appears to be involved in downregulation of the activity of cyclooxigenase 2 (COX2) and nuclear factor kappa-b $(\mathrm{NF} \kappa \mathrm{B})$. Likewise, PPAR $\gamma$ exhibits anti-inflammatory properties. It is upregulated in activated macrophages and inhibits 
the expression, for example, of the inducible nitric oxide synthase [6].

Genetic polymorphisms of PPAR have been investigated in numerous conditions, predominantly in metabolic disorders like type 2 diabetes or atherosclerosis [7-9]. There is evidence for accelerated atherosclerosis in WG patients [10], suggesting that both conditions may share some (genetic) risk factors.

Given the pleiotropic role of PPAR in the regulation of (vascular) inflammation, we have hypothesised that genetic predisposition to WG may arise from the interaction of certain genetic variations of RXRB and PPAR. We have, therefore, screened the $R X R B$ gene for novel, potentially WGspecific variations. Moreover, we have genotyped two single nucleotide polymorphisms in each of the genes for PPAR $\alpha$ and PPAR $\gamma$ in large panels of WG cases and healthy controls. Finally, we searched for epistatic effects of certain alleles of $R X R B$ and PPARA or PPARG.

\section{Patients and Methods}

2.1. Subjects. All patients included in this study were diagnosed with WG according to the criteria of the American College of Rheumatology and the Chapel Hill consensus conference [11]. They were selected at the interdisciplinary vasculitis centre at the University of Luebeck/Rheumaklinik Bad Bramstedt. All patients were asked for their ancestry and reported German decent for at least two generations. Healthy German blood donors were used as controls and ancestry was evaluated equal to the patient group.

For the RXRB locus we genotyped the same patients $(n=$ $282)$ and controls $(n=380)$ that were previously analyzed for the $6 \mathrm{p} 21.3$ locus [3]. In order to increase statistical power, the SNP in the PPAR genes were analyzed in expanded sample comprising 462 WG cases and 701 controls.

Ethical principles for medical research involving human subjects as defined in the Declaration of Helsinki have been followed. The study design was approved by the local ethics committee at the University of Luebeck, Germany (No. AZ 06-087).

2.2. Genotyping. Four types SNPs rs9277935, rs2072915, rs2744537, and rs1547387 (see Figure 1) were selected from the HapMap database to serve as tagSNPs for a region of $10 \mathrm{~kb}$ including the RXRB gene. With this selection all HapMap SNPs within this region are efficiently tagged $\left(r^{2}>0.9\right)$. rs2072915 and $r s 2744537$ are located within the $3^{\prime}$ untranslated region (UTR) of $R X R B$, while rs 9277935 is located approximately $1 \mathrm{~kb} 3^{\prime}$ of the last $R X R B$ exon. rs 1547387 is located $5^{\prime}$ of RXRB, in exon 5 of the SLC39A7 gene, in which it constitutes a silent SNP (Ser209Ser).

rs9277935, rs2072915, and rs2744537 were genotyped via PCR-RFLP techniques and a commercially available TaqMan genotyping assay (Applied Biosystems) was used for rs1547387.

For rs1800206 (Val162Leu) in PPARA also a commercially available TaqMan assay was used, while rs6008259 (3' UTR of PPARA), rs1801282 (PPARG, Ala12Pro), and

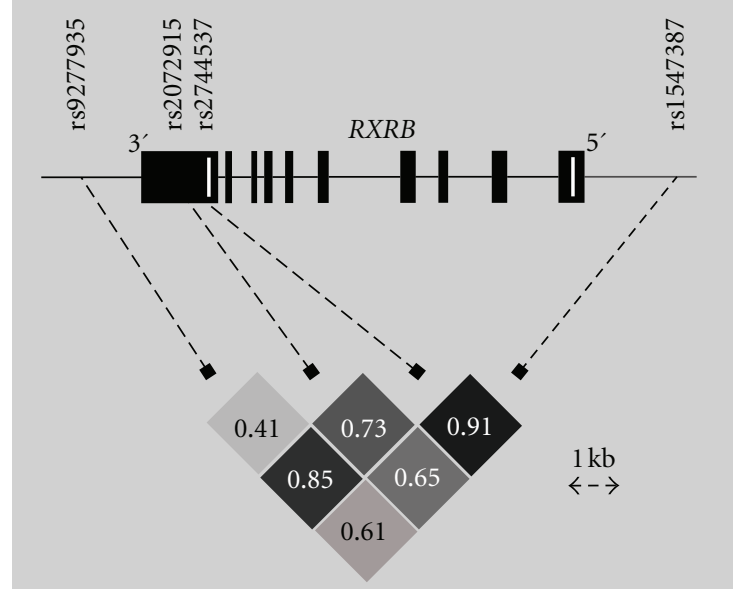

FIGURE 1: Location of the four tagging SNPs of the RXRB gene and $\mathrm{LD}$ structure. The $\mathrm{LD}$ is plotted as $D^{\prime}$ and intensity coded (darker shades corresponding to higher degree of LD). The RXRB gene is transcribed from centromer to telomer, that is, from right to left. $R X R B$ exons are depicted as black boxes, and translation start and stop points are marked by white vertical lines.

rs3856806 (PPARG, His449His) were genotyped using newly designed primers and probes (see Table 1).

2.3. Direct Sequencing of RXRB. The entire RXRB gene (all 10 exons and exon/intron boundaries) was directly sequenced in 5 WG patients typed homozygous for the associated $R X R B$ haplotype (see below; primer sequences available on request). For comparison 5 healthy controls homozygous for the inversely associated (i.e., protective) haplotype were also sequenced.

2.4. Statistical Analysis. Genotypes were recorded in linkage format. Association for each single marker was tested by using chi square tests on contingency tables. A $P$ value $<.05$ was considered significant. LD between each SNP of a respective locus, and haplotype block frequencies were calculated by using Haploview 4.1 [12] .

Interaction between $R X R B$ and PPARA/PPARG SNPs were calculated with GAIA (http://gump.qimr.edu.au/GAIA/ gaia.html; [13]). This application uses a logistic regression model which tests for pairwise locus/locus interactions between genes. We applied an additive interaction model for each pair of SNPs testing the significance of the interaction model terms over and above any main effects.

\section{Results and Discussion}

The overall call rate for all 8 SNPs in patients and controls was $93.60 \%$. None of the SNPs revealed significant deviation from Hardy-Weinberg equilibrium. Two of the RXRB SNPs (rs9277935 and rs2744537) showed highly significant association with WG (see Table 2). Accordingly, the GTTC haplotype (calculated from all four SNPs of this locus) was significantly more common in WG while the TTGC haplotype was overrepresented in controls (see Table 2). 
TABLE 1: Oligonucleotides and restriction enzymes used for genotyping.

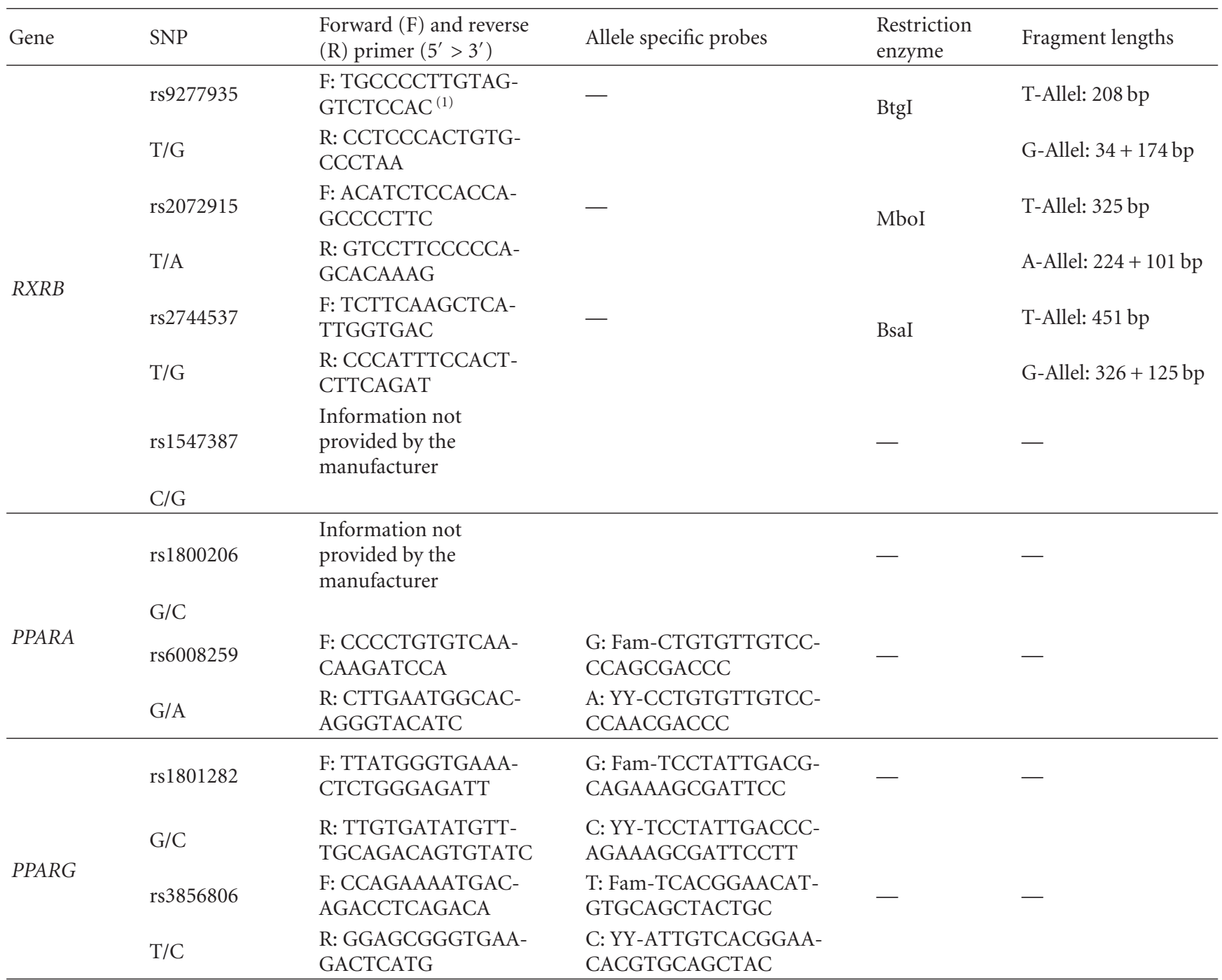

(1) A 17 mer nucleotide (GTAAAACGACGGCCAGT) was added $5^{\prime}$ to the forward primer increase fragment length differences between the two alleles, FAM: 6-carboxy-fluorescein; YY: Yakima Yellow.

Based on the SNP data we have then selected 5 WG patients homozygous for the GTTC haplotype for direct sequencing of the RXRB gene. For comparison 5 control subjects homozygous for the TTGC haplotype were also analyzed. Yet, no novel sequence variation was detected, a finding which is consistent with previous results from Szyld et al. [4], who had screened the RXRB gene in WG patients without consideration of the haplotype structure of this locus. Results from the initial tagging SNP genotyping were confirmed in the ten included individuals for the two exonic tagging SNPs (rs2744537 and rs2072915). Two other exonic SNPs (rs1152296 in exon 10/5'UTR and rs6531 in exon 7) were identified and perfectly segregated with the previously identified risk/nonrisk haplotypes.

Both associated RXRB SNPs (rs9277935 and rs2744537) are in strong LD with HLA-DPB1 as well as with rs3117228 and rs213208 (see Figure 2) which had revealed the highest (and partly HLA-DPB1 independent) association with WG in a previous study [3]. Moreover, according to the HapMap data rs2744537 is in strong LD with rs6531 (a synonymous SNP in exon 7 of $R X R B$ ) which was significantly associated with WG earlier [4]. As for the other associated SNPs in this genomic region, it is therefore hard to differentiate which of the SNPs (or even a yet unknown variation of this locus) constitutes the primary WG risk factor. A potential approach to overcome this problem is the analysis of factors related to the different proteins encoded in the $6 \mathrm{p} 21.3$ region. We have therefore investigated SNPs in the genes for PPAR $\alpha$ and $\operatorname{PPAR} \gamma$ which are functionally closely linked to RXRB.

The two SNPs in PPARG were in weak to moderate LD $\left(D^{\prime}=0.64, r^{2}=0.40\right)$ while the two SNPs in PPARA were virtually unlinked $\left(D^{\prime}=0.47, r^{2}=0.06\right)$. Therefore, no haplotype frequencies were calculated for these genes. Both SNPs in PPARG showed very similar allele frequencies in cases and controls not revealing any significant differences (see Table 2). The two SNPs in PPARA both revealed a 
TABLE 2: Allele and haplotype frequencies.

\begin{tabular}{|c|c|c|c|c|c|c|}
\hline Gene & SNP/haplotype & Allele & Frequency in controls & Frequency WG patients & $P$ value & OR $(95 \% \mathrm{CI})$ \\
\hline \multirow{8}{*}{$R X R B$} & \multirow{2}{*}{ rs9277935 } & G & 0.76 & 0.89 & $6.65 \times 10^{-9}$ & $2.55(1.86-3.56)$ \\
\hline & & $\mathrm{T}$ & 0.24 & 0.11 & & \\
\hline & \multirow{2}{*}{ rs2072915 } & $\mathrm{T}$ & 0.71 & 0.75 & .157 & $1.20(0.93-1.55)$ \\
\hline & & A & 0.29 & 0.25 & & \\
\hline & \multirow{2}{*}{ rs2744537 } & $\mathrm{T}$ & 0.73 & 0.57 & $6.63 \times 10^{-9}$ & $0.49(0.39-0.63)$ \\
\hline & & G & 0.27 & 0.43 & & \\
\hline & \multirow{2}{*}{ rs 1547387} & $\mathrm{C}$ & 0.87 & 0.85 & .34 & $0.85(0.62-1.18)$ \\
\hline & & G & 0.13 & 0.15 & & \\
\hline \multirow{3}{*}{$R X R B^{*}$} & GTTC & & 0.24 & 0.40 & $1.15 \times 10^{-9}$ & $2.11(1.66-2.69)$ \\
\hline & TTGC & & 0.19 & 0.07 & $9.73 \times 10^{-10}$ & $0.32(0.22-0.47)$ \\
\hline & other & & 0.43 & 0.47 & .157 & $1.18(0.94-1.47)$ \\
\hline \multirow{4}{*}{ PPARA } & \multirow{2}{*}{ rs1800206 } & $\mathrm{C}$ & 0.93 & 0.95 & .076 & $1.40(0.96-2.05)$ \\
\hline & & G & 0.07 & 0.05 & & \\
\hline & \multirow{2}{*}{ rs6008259 } & G & 0.80 & 0.83 & .069 & $0.81(0.65-1.02)$ \\
\hline & & A & 0.20 & 0.17 & & \\
\hline \multirow{4}{*}{ PPARG } & \multirow{2}{*}{ rs1801282 } & $\mathrm{C}$ & 0.85 & 0.85 & .74 & $0.96(0.75-1.23)$ \\
\hline & & G & 0.15 & 0.15 & & \\
\hline & \multirow{2}{*}{ rs3856806 } & $\mathrm{C}$ & 0.86 & 0.85 & .70 & $0.95(0.75-1.22)$ \\
\hline & & $\mathrm{T}$ & 0.14 & 0.15 & & \\
\hline
\end{tabular}

OR: Odds ratio,

CI: confidence interval,

*haplotype frequencies calculated from all four RXRB SNPs.

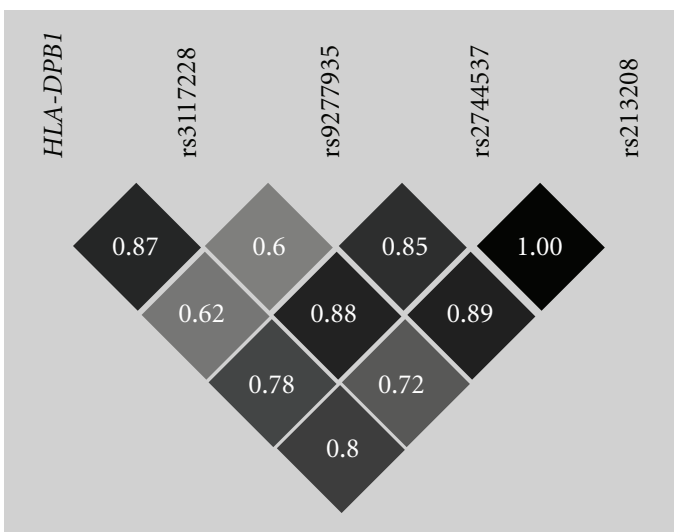

Figure 2: LD between WG associated variations of the $6 \mathrm{p} 21.3$ genomic region. HLA-DPB1 is modelled as a biallelic marker with the associated $H L A-D P B 1^{*} 0401$ as one allele and all other alleles joint into the other allelic category. LD is plotted as $D^{\prime}$ and intensity coded (darker shades corresponding to higher degree of LD). Both RXRB SNPs investigated in this study (rs9277935 and rs2744537) are in high LD with $H L A-D P B 1$ as well as with the markers analyzed by Heckmann et al. (2008): rs3117228 ( $3^{\prime}$ of HLA-DPB1) and rs213208 (intronic SNP in RING1, approx. $10 \mathrm{~kb}$ centromeric of $R X R B)$.

decreased frequency of the minor allele but these differences did not reach significance level (see Table 2). No significant pairwise epistatic effect for any of the investigated SNPs, that is, neither between PPARA and PPARG nor between PPARA (or PPARG, resp.) and RXRB or HLA-DPB1. Taken together, we cannot provide evidence for a genetically based involvement of PPAR $\alpha$ and PPAR $\gamma$ in the etiopathology of WG. Moreover, the strong WG association with the 6p21.3 locus is unlikely to be based on any coding variation of the $R X R B$ gene. Future studies will therefore have to focus on regulatory elements of this area (e.g., cis acting elements or micro RNAs).

\section{Conclusions}

These results do not support a direct etiopathological role of RXRB and/or PPAR in WG. Analyses of further genes functionally linked to RXRB, for example, retinoic acid receptors or vitamin $\mathrm{D}$ receptors, may provide additional data useful to evaluate the RXRB association found in WG.

\section{Acknowledgment}

This work was supported by the Deutsche Forschungsgemeinschaft [KFO 170].

\section{References}

[1] E. Csernok, F. Moosig, and W. L. Gross, "Pathways to ANCA production: from differentiation of dendritic cells by proteinase 3 to $\mathrm{B}$ lymphocyte maturation in Wegener's 
granuloma," Clinical Reviews in Allergy \& Immunology, vol. 34, no. 3, pp. 300-306, 2008.

[2] P. Jagiello, M. Gencik, L. Arning, et al., "New genomic region for Wegener's granulomatosis as revealed by an extended association screen with 202 apoptosis-related genes," Human Genetics, vol. 114, no. 5, pp. 468-477, 2004.

[3] M. Heckmann, J. U. Holle, L. Arning, et al., “The Wegener's granulomatosis quantitative trait locus on chromosome 6p21.3 as characterised by tagSNP genotyping," Annals of the Rheumatic Diseases, vol. 67, no. 7, pp. 972-979, 2008.

[4] P. Szyld, P. Jagiello, E. Csernok, W. L. Gross, and J. T. Epplen, "On the Wegener granulomatosis associated region on chromosome 6p21.3," BMC Medical Genetics, vol. 7, article 21, pp. 1-11, 2006.

[5] P. R. Devchand, H. Keller, J. M. Peters, M. Vazquez, F. J. Gonzalez, and W. Wahli, "The PPAR $\alpha$-leukotriene $\mathrm{B}_{4}$ pathway to inflammation control," Nature, vol. 384, no. 6604, pp. 3943, 1996.

[6] M. Ricote, A. C. Li, T. M. Willson, C. J. Kelly, and C. K. Glass, "The peroxisome proliferator-activated receptor- $\gamma$ is a negative regulator of macrophage activation," Nature, vol. 391, no. 6662, pp. 79-82, 1998.

[7] K. A. Volcik, J. A. Nettleton, C. M. Ballantyne, and E. Boerwinkle, "Peroxisome proliferator-activated receptor $\alpha$ genetic variation interacts with n-6 and long-chain $n-3$ fatty acid intake to affect total cholesterol and LDL-cholesterol concentrations in the Atherosclerosis Risk in Communities Study," The American Journal of Clinical Nutrition, vol. 87, no. 6, pp. 1926-1931, 2008.

[8] L. Dongxia, H. Qi, L. Lisong, and G. Jincheng, "Association of peroxisome proliferator-activated receptor $\gamma$ gene Pro12Ala and C161T polymorphisms with metabolic syndrome," Circulation Journal, vol. 72, no. 4, pp. 551-557, 2008.

[9] M.-J. Shin, A. M. Kanaya, and R. M. Krauss, "Polymorphisms in the peroxisome proliferator activated receptor $\alpha$ gene are associated with levels of apolipoprotein CIII and triglyceride in African-Americans but not Caucasians," Atherosclerosis, vol. 198, no. 2, pp. 313-319, 2008.

[10] K. de Leeuw, J.-S. Sanders, C. Stegeman, A. Smit, C. G. Kallenberg, and M. Bijl, "Accelerated atherosclerosis in patients with Wegener's granulomatosis," Annals of the Rheumatic Diseases, vol. 64, no. 5, pp. 753-759, 2005.

[11] J. C. Jennette, R. J. Falk, K. Andrassy, et al., "Nomenclature of systemic vasculitides: proposal of an international consensus conference," Arthritis \& Rheumatism, vol. 37, no. 2, pp. 187$192,1994$.

[12] J. C. Barrett, B. Fry, J. Maller, and M. J. Daly, "Haploview: analysis and visualization of LD and haplotype maps," Bioinformatics, vol. 21, no. 2, pp. 263-265, 2005.

[13] S. Macgregor and I. A. Khan, "GAIA: an easy-to-use webbased application for interaction analysis of case-control data," BMC Medical Genetics, vol. 7, article 34, pp. 1-6, 2006. 


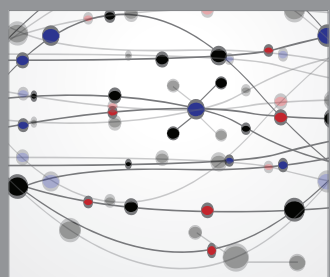

The Scientific World Journal
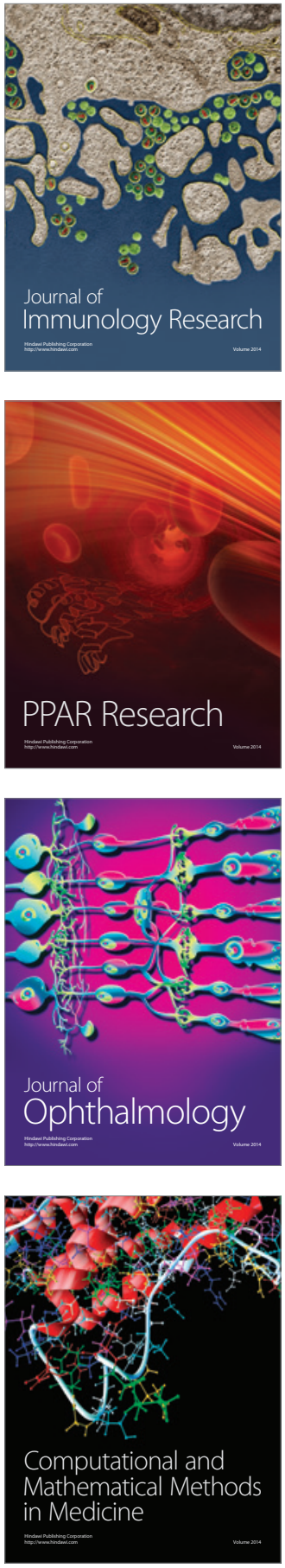

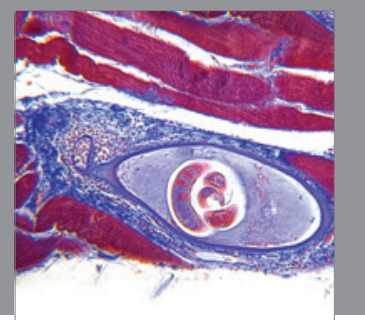

Gastroenterology

Research and Practice
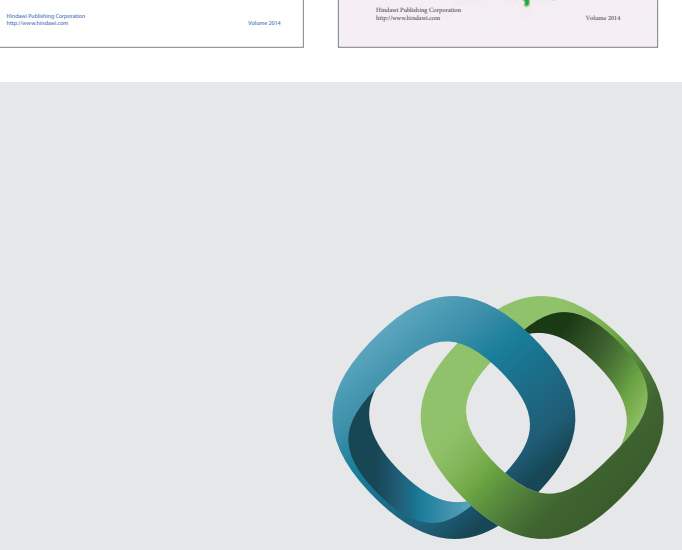

\section{Hindawi}

Submit your manuscripts at

http://www.hindawi.com
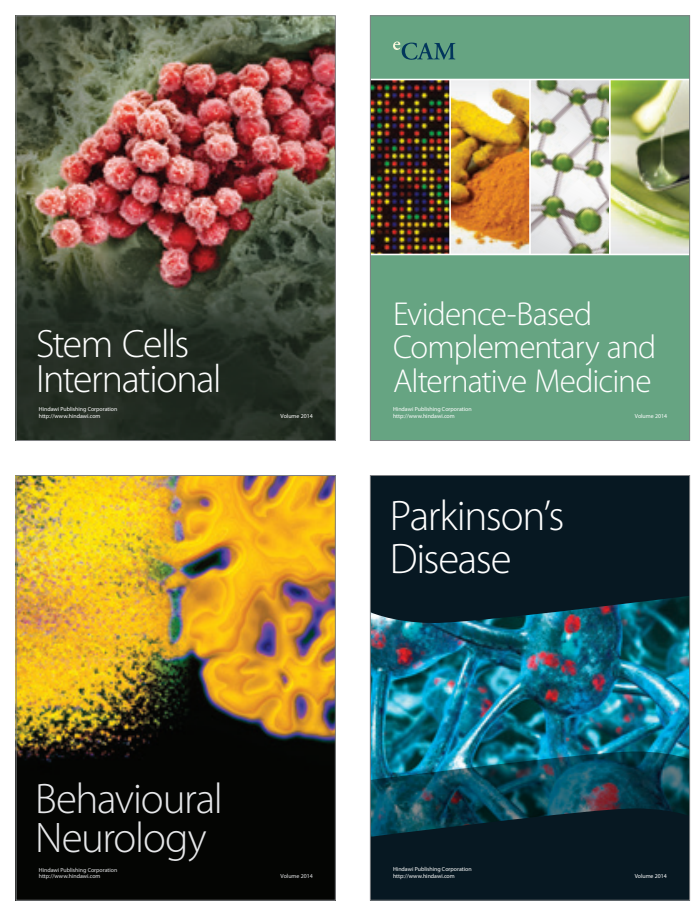

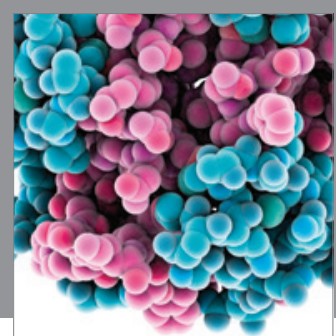

Journal of
Diabetes Research

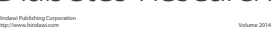

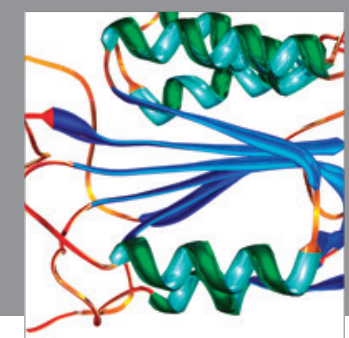

Disease Markers
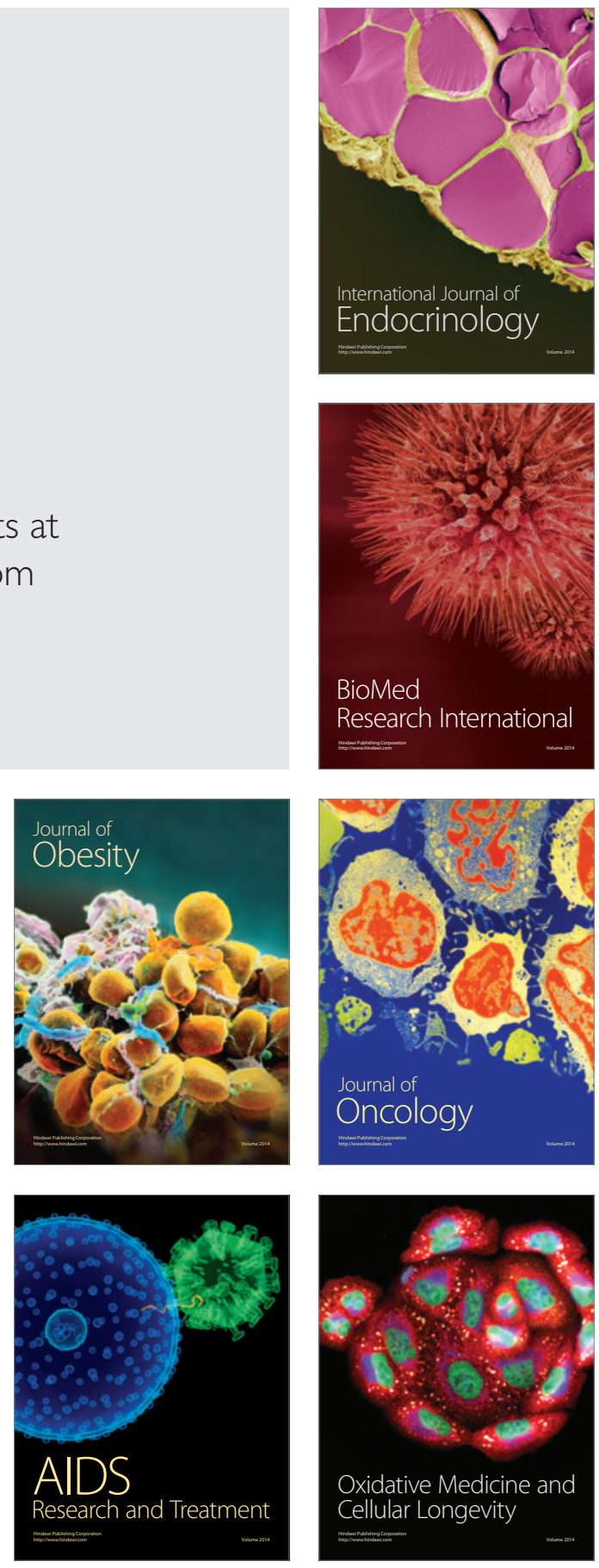